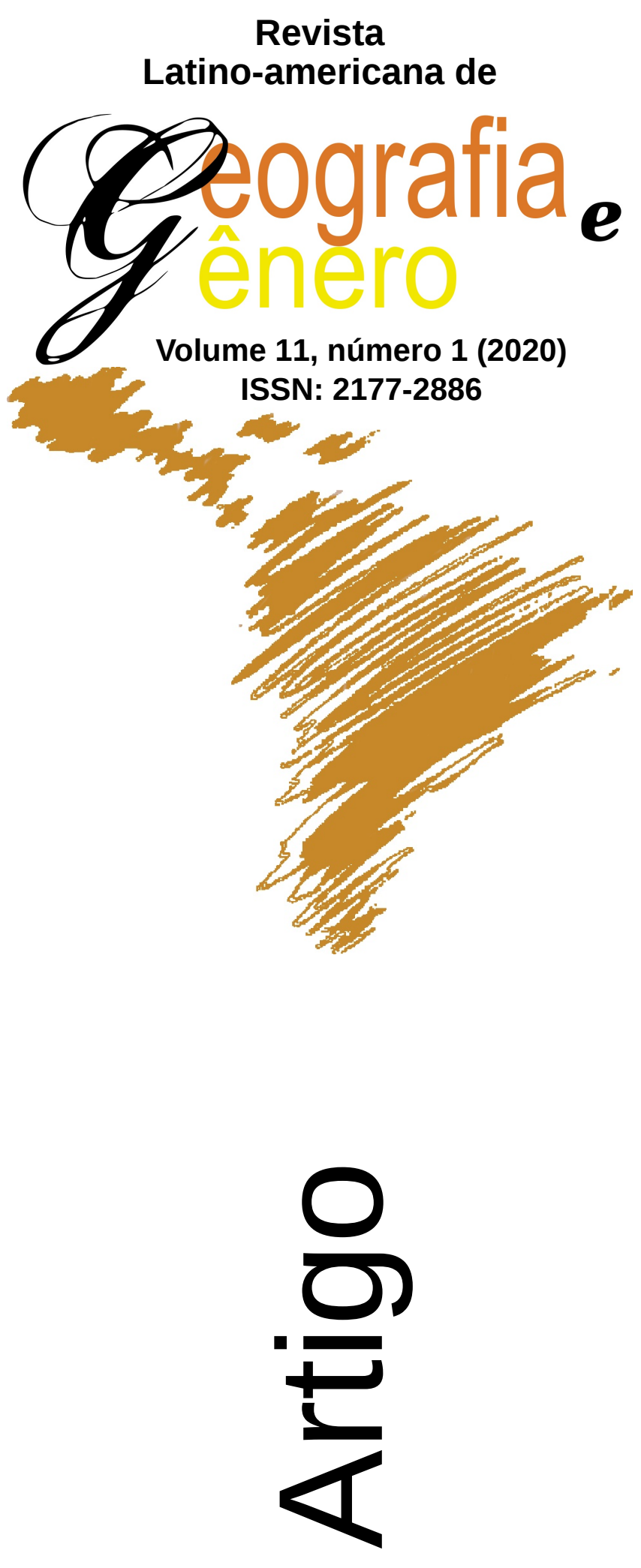

Representaciones Sociales sobre Acoso Sexual Callejero en Mujeres Activistas Participantes del XXXIII Encuentro Nacional de Mujeres, Argentina

Representações Sociais sobre o Assédio Sexual de Rua nas Mulheres Participantes do XXXIII Encuentro Nacional de Mujeres, Argentina

Social Representations about Etreet Sexual Harassment in Women Activists Participating in the XXXIII National Meeting of Women, Argentina

Almendra Aladro

Universidad Nacional de Mar del Plata - Argentina almendraaladro@gmail.com

Como citar este artigo:

ALADRO, Almendra. Representaciones Sociales sobre Acoso Sexual Callejero en Mujeres Activistas Participantes del XXXIII Encuentro Nacional de Mujeres, Argentina. Revista Latino Americana de Geografia e Gênero, v. 11, n. 1, p. 113 - 133, 2020. ISSN 2177-2886.

Disponível em:

http://www.revistas2.uepg.br/index.php/rlagg 


\title{
Representaciones Sociales sobre Acoso Sexual Callejero en Mujeres Activistas Participantes del XXXIII Encuentro Nacional de Mujeres, Argentina
}

\author{
Representações Sociais sobre o Assédio Sexual de Rua nas Mulheres \\ Participantes do XXXIII Encuentro Nacional de Mujeres, Argentina
}

\section{Social Representations about Etreet Sexual Harassment in Women Activists Participating in the XXXIII National Meeting of Women, Argentina \\ Resumen}

En este trabajo, se presentan y discuten los resultados de un estudio descriptivo de corte transversal en el cual se aplicaron cuestionarios cerrados a una población de 44 mujeres participantes del Taller No43 "Mujeres y Hábitat", en el marco del XXXIII Encuentro Nacional de Mujeres (Argentina) desarrollado entre el 13 y el 15 de octubre de 2018 en la provincia de Chubut, el cual tuvo como objetivos identificar: 1) la conceptualización predominante del acoso sexual callejero y su correlación con las definiciones establecidas tanto en leyes vigentes como en proyectos de ley de índole provincial; 2) las respuestas formales exigidas al Estado; y 3) las medidas a adoptar frente a hechos de acoso sexual callejero en relación al victimario. Se planteó un muestreo no probabilístico de tipo casual, por ende, carente de representatividad. Se suministró a las participantes un cuestionario consistente de tres preguntas de carácter demográfico (edad, provincia y ciudad de origen e identidad de género), y cinco preguntas cerradas que tuvieron por objetivo la evaluación de: 1) el tipo de definición sobre acoso sexual callejero predominante; 2) la consideración del fenómeno como un problema de seguridad; 3) la existencia de una exigencia de intervención del Estado en la cuestión; 4) el tipo de medidas que se demandan al Estado, en caso de considerarse necesaria su intervención; y 5) el tipo de sanción necesaria para los victimarios, en caso de ser preferida una respuesta punitiva de índole contravencional y/o penal por parte del Estado.

Palabras-Clave: Acoso Sexual Callejero; Encuentro Nacional de Mujeres, Hábitat; Ciudad; Políticas Públicas.

\section{Resumo}

Este trabalho discute os resultados de um estudo descritivo de corte transversal no qual foram aplicados questionários fechados a uma população de 44 mulheres participantes do Taller $n .^{\circ}$ 43 'Mujeres y Hábitat' do XXXIII Encuentro Nacional de Mujeres, Argentina, ocorrido entre os dias 13 a 15 de outubro de 2018 em 'Chubut', que teve o objetivo de identificar (1) a conceituação predominante do assédio sexual de rua e sua correção com as definições estabelecidas tanto nas leis vigentes como no projeto de lei da provincia, (2) as respostas formais exigidas do Estado e (3) as medidas a serem adotadas frente aos atos de assédio sexual de rua em relação as vítimas. A amostra foi não probabilístico de tipo casual, por isto carecendo de representatividade. Foi ministrado as participantes um questionário que consistiu em 3 perguntas de carácter demográfico (idade, província, cidade de origem e identidade de gênero) e 5 perguntas fechadas que tiveram por objetivo a avaliação do (1) tipo de definição sobre o assédio sexual de rua predominante, (2) a consideração do fenômeno como um problema de segurança, (3) a existência de uma exigência de interveção do Estado na quesțão, 
(4) o tipo de medidas que se demandam do Estado, no caso de se considerar necessária sua intervenção, e (5) o tipo de sansão necesária para as vítimas, no caso de ser preferida uma resposta punitiva de índole contravencional e/ou penal por parte do Estado.

Palavras-Chave: Assédio Sexual na Rua; Encontro Nacional de Mulheres, Habitat; Cidade; Políticas públicas.

This paper presents the results of a descriptive cross-sectional study in which closed questionnaires were supplied to a population of 44 women participants of Workshop No.43, "Women and Habitat", of the XXXIII National Meeting of Women (Argentina), held on July 13th-15th at Chubut, that aimed to identify 1) the predominant conceptualization of street sexual harassment and its correlation with the definitions established both in current laws and in provincial bills; 2) the formal responses required from the State; and 3) the measures to be adopted in the face of acts of sexual street harassment in relation to the victimizer. The sampling was non-probability of the casual type, therefore it will lack representativeness. Participants were given a questionnaire consisting of three demographic questions (age, province and city of origin, and gender identity) and five closed questions that were aimed at evaluating 1) the definition of the predominant street sexual harassment; 2) the consideration degree of the phenomenon as a security problem; 3 ) the existence of a requirement for State intervention in the matter; 4) the type of measures that are demanded from the State, should its intervention be considered necessary; and 5) the type of sanction that should be imposed on the perpetrators if it is considered as a contravention of the law or as a criminal act that requires a punitive response from the State.

Keywords: Street Sexual Harassment; National Meeting of Women, Habitat; City; Public politics.

\section{Introducción}

A partir del internacionalmente reconocido '\#NiUnaMenos', el movimiento de mujeres en Argentina se posicionó desde el año 2015 a la vanguardia de la lucha contra el patriarcado, gestando acciones y contenidos tomados como modelos por las feministas de otros países. No obstante, la organización feminista en este país es de larga data, siendo el hito fundacional de una agenda común y trasversal el I Encuentro Nacional de Mujeres en el año 1986 en la ciudad de Buenos Aires, evento que lleva a la fecha treinta y tres ediciones ininterrumpidas.

El Encuentro Nacional de Mujeres $^{1}$ es un evento autoconvocado y autogestionado que se lleva a cabo anualmente y que invita a todas las mujeres, independientemente de la pertenencia política o partidaria, a debatir de manera democrática, horizontal y consensuada los tópicos principales de la agenda feminista nacional. Los intercambios se realizan en formato taller, los cuales incluyen diferentes temáticas vinculadas a la realidad de las mujeres y que finalizan con la redacción conjunta de las conclusiones arribadas en un documento. Esas conclusiones sintetizan las luchas y preocupaciones que el movimiento feminista viene planteando desde los distintos lugares de inserción de trabajo o de estudio que las mujeres participantes ocupan.

Pese a su realización ininterrumpida, los tópicos abordados durante los

1 A partir del año 2019 se realiza bajo el nombre 'Encuentro Plurinacional de Mujeres, Lesbianas, Trans, Travestis, Bisexuales y No Binaries', pero el abordaje del proceso político que dio lugar al cambio excede el objeto de este trabajo. 
encuentros no han sido los mismos con el paso del tiempo, lo cual se ha visto representado en el aumento de la cantidad de talleres programados y en las conclusiones que de los mismos surgen.

Los debates en torno a la ciudad y el hábitat están instalados como taller permanente bajo el nombre de 'Mujer y Hábitat'. En el Encuentro del año 2018, el temario de este taller fue: 1) Derecho a la tierra, la vivienda y los servicios; 2) Luchas por la vivienda, el barrio, los servicios y el equipamiento comunitario; 3) Tomas, ocupaciones, asentamientos; 4) El espacio público y privado: desigualdades y diferencias en el uso del espacio urbano; 5) Desalojos; 6) El rol del Estado en las políticas públicas y la planificación urbana; 7) La participación de las mujeres en la construcción del hábitat; 8) Análisis del espacio público desde una perspectiva de género; 9) Gestión de un medio ambiente saludable; 10) Ciudades seguras, inclusivas y sin violencia hacia las mujeres; 11) Participación de las mujeres en la elaboración de políticas urbanas; 12) Intervenciones y experiencias.

El acoso sexual callejero, como forma de violencia específica basada en género en contextos urbanos, ha sido un tema de agenda política estudiado tanto por los movimientos feministas como por organismos públicos. Siendo el intento de solución que ha primado en la incorporación de figuras de índole contravencional a numerosas normativas provinciales (ALADRO, 2017), mientras que, a nivel federal, el proyecto de ley que pretende configurarlo como un delito penal contra la integridad sexual ${ }^{2}$ ya cuenta con media sanción de la Cámara de Diputados de la Nación.

Dada las especificidades de las temáticas abordadas en la edición trigésimo tercera del Encuentro y la incipiencia de la problematización social del acoso sexual callejero, se ha decidido realizar un estudio de carácter mixto, tomando como población a las participantes del Taller 'Mujeres y Hábitat' del XXXIII Encuentro Nacional de Mujeres, el cual tiene los objetivos de identificar: 1) la conceptualización predominante del acoso sexual callejero y su correlación con las definiciones establecidas tanto en leyes vigentes como en proyectos de ley de índole provincial; 2) las respuestas formales exigidas al Estado; y 3) las medidas a adoptar frente a hechos de acoso sexual callejero en relación al victimario.

El objetivo de este artículo es presentar los resultados del estudio realizado, para lo cual se dividirá la exposición en cuatro acápites, a saber: 1) estado de la cuestión, a los fines de explorar la bibliografía existente respecto del Encuentro Nacional de Mujeres y del acoso sexual callejero como objetos de estudio; 2) materiales y métodos, donde se detallarán las especificidades del estudio realizado; 3) resultados, en el que se expondrán los productos; y 4) discusión, donde se pondrá en diálogo a los resultados obtenidos con distintos hechos y saberes.

\section{Breve marco teórico y estado de la cuestión}

\section{Las representaciones sociales}

Gladys Villarroel ha estudiado el contexto de producción del concepto de representación social dando cuenta del dinamismo e innovación que este

2 Cámara de Diputados de la Nación, Expediente 2405-D-2015. 
término encierra. Así, define las representaciones sociales como "modalidades específicas de conocimiento del sentido común que se construyen en los intercambios de la vida cotidiana" e identifica, como sus notas características, su producción colectiva y su origen en "la intersección entre los psicológico y lo social" (VILLARROEL, 2007, p. 434).

Esta definición es doblemente útil a los fines de este trabajo. Por un lado, las participantes del estudio, en tanto mujeres, han construido nociones y explicaciones del acoso sexual callejero por haberlo recibido en algún momento de sus vidas. Por el otro, en tanto sujetas organizadas, dan un contenido semántico conscientemente politizado a la problemática aquí estudiada buscando explicaciones a su origen histórico y dando fundamentos a la demanda colectiva de su erradicación.

\section{Género, ciudad y acoso sexual callejero}

El hito iniciático en la elaboración del concepto de derecho a la ciudad es la obra de Henri Lefebvre que, precisamente, se titula "El derecho a la ciudad". En ella se sostiene como una de sus premisas centrales que:

Lo urbano (...) es el resultado de una historia, de una praxis social, de personas y grupos determinados que realizan esta obra en condiciones históricas (...)" y, así, “(...)la ciudad como obra, como resultado de esta praxis, así la vida cotidiana se torna obra, apropiación del espacio, uso (LEFEBVRE, 1967, p. 68).

Explica Ana Falú que "las mujeres permanecerán invisibles en las ciudades, relegadas al ámbito privado del hogar, lo que tendrá sustento en la fuerte división del trabajo sexual imperante" (FALÚ, 2016, p. 160), afirmación en la que se encuentra latente la dicotomía público/privado como eje estructurante de las desigualdades urbanas basadas en género. En este razonamiento, "las ciudades no son percibidas ni usadas de la misma manera por hombres y mujeres" ya que las mujeres "acceden a los bienes y servicios públicos de manera distinta, combinando trabajo productivo y reproductivo" (FALÚ, 2016, p. 163).

El acoso sexual callejero, en este orden de ideas, puede entenderse como una de las consecuencias de la contradicción que implica que las mujeres ocupen el espacio público que ha sido producido por y para varones, desde el inicio de las ciudades como las conocemos. Este tipo de conductas están reconocidas en el 'artículo 6 de la Ley 26495' como modalidad de violencia hacia la mujer desde el 16 de abril de 2019 como:

(...) aquella ejercida contra las mujeres por una o más personas, en lugares públicos o de acceso público, como medios de transporte o centros comerciales, a través de conductas o expresiones verbales o no verbales, con connotación sexual, que afecten o dañen su dignidad, integridad, libertad, libre circulación o permanencia y/o generen un ambiente hostil u ofensivo (...).

Los estudios en la materia se centran en el análisis de las representaciones sociales respecto del fenómeno por mujeres jóvenes en ciudades determinadas (CORAZÓN LLERENA BENITES, 2016; MEDINA VILCA CASTRO Y 
ANAIZ, 2016), su relación con la seguridad ciudadana (MORALES ALFONSO, QUIROZ DEL POZO Y RAMÍREZ IGLESIAS, 2016), su naturaleza como violencia urbana basada en género (FALÚ, 2017) y la existencia de dispositivos normativos locales que sancionen la problemática (ALADRO, 2017; GALVAÑO TER-AKOPIÁN, 2020).

\section{El Encuentro Nacional de Mujeres como objeto de estudio}

Los trabajos académicos que toman el fenómeno del Encuentro Nacional de Mujeres en Argentina son escasos y apuntan tanto al abordaje de sus generalidades (ALMA \& LORENZO, 2009: ANGILLETTA, 2013; FERNÁNDEZ VÁZQUEZ, 2016; HERRERA, 2017), como a temáticas específicas, a saber: la participación de mujeres indígenas (SCIORTINO, 2014; 2017), representaciones sociales respecto de la laicidad (FELITTI, 2017), el reconocimiento de la prostitución como trabajo sexual (MARTINOWSKYVJ, 2017), las relaciones entre los Estados provinciales y el movimiento de mujeres (GARCÍA, 2013), y construcción del hábitat (ALADRO, 2016; 2017).

\section{Materiales y métodos}

El estudio cuyos resultados aquí se presentan es de tipo descriptivo de corte transversal. De una población estimada por la Comisión Organizadora de 50000 mujeres asistentes al XXXIII Encuentro Nacional de Mujeres de las cuales 112 participaron del Taller No43 'Mujeres y Hábitat', se obtuvo una muestra de 44 mujeres. El muestreo fue no probabilístico de tipo casual, por ello carecerá de representatividad.

Para la recolección de datos se requirió autorización de la moderadora del taller y de la Comisión Organizadora del Encuentro, lo cual llevó a que la actividad pudiera desarrollarse recién el segundo día de talleres en el horario de 9 a 12 hrs. Se suministró a las participantes un cuestionario consistente en 3 preguntas de carácter demográfico (edad, provincia y ciudad de origen e identidad de género) y cinco preguntas cerradas que tuvieron por objetivo la evaluación de: 1) el tipo de definición sobre acoso sexual callejero predominante; 2) la consideración del fenómeno como un problema de seguridad; 3) la existencia de una exigencia de intervención del Estado en la cuestión; 4) el tipo de medidas que se demandan al Estado, en caso de considerarse necesaria su intervención; y 5) el tipo de sanción necesaria para los victimarios, en caso de ser preferida una respuesta punitiva de índole contravencional y/o penal por parte del Estado.

Para la validación del instrumento se realizaron consultas con pares investigadores formados en metodología de la investigación, quienes realizaron observaciones a la formulación inicial de las preguntas que permitieron generar un diseño final del instrumento claro, específico $\mathrm{y}$ fácilmente procesable.

El procesamiento de datos se realizó a través del programa Microsoft Office Excel 2007, mediante estadística descriptiva y porcentajes para evaluar el tipo grado de preferencias de las participantes respecto de las respuestas preestablecidas, su relación con las variables demográficas y las relaciones que pueden establecerse entre preferencias. 


\section{Resultados}

Respecto al análisis de los datos demográficos brindados por las 44 participantes, destacaremos los más representativos. Así, a modo de caracterización de la muestra se desprende que el 100\% de ellas eran mujeres cisgénero, de las cuales $60,46 \%$ tenían entre 16 y 30 años; $20,93 \%$ entre 31 y 45 años; y 18,61\% 46 años o más (Figura 1). Respecto a la provincia de procedencia, 36,36\% eran de Buenos Aires; 34,88\% de Ciudad Autónoma de Buenos Aires (en adelante, CABA); $11,63 \%$ de Chubut; y 4,65\% tanto de Río Negro como de Santa Fe (Figura 2).

Figura 1: Datos demográficos (edad)

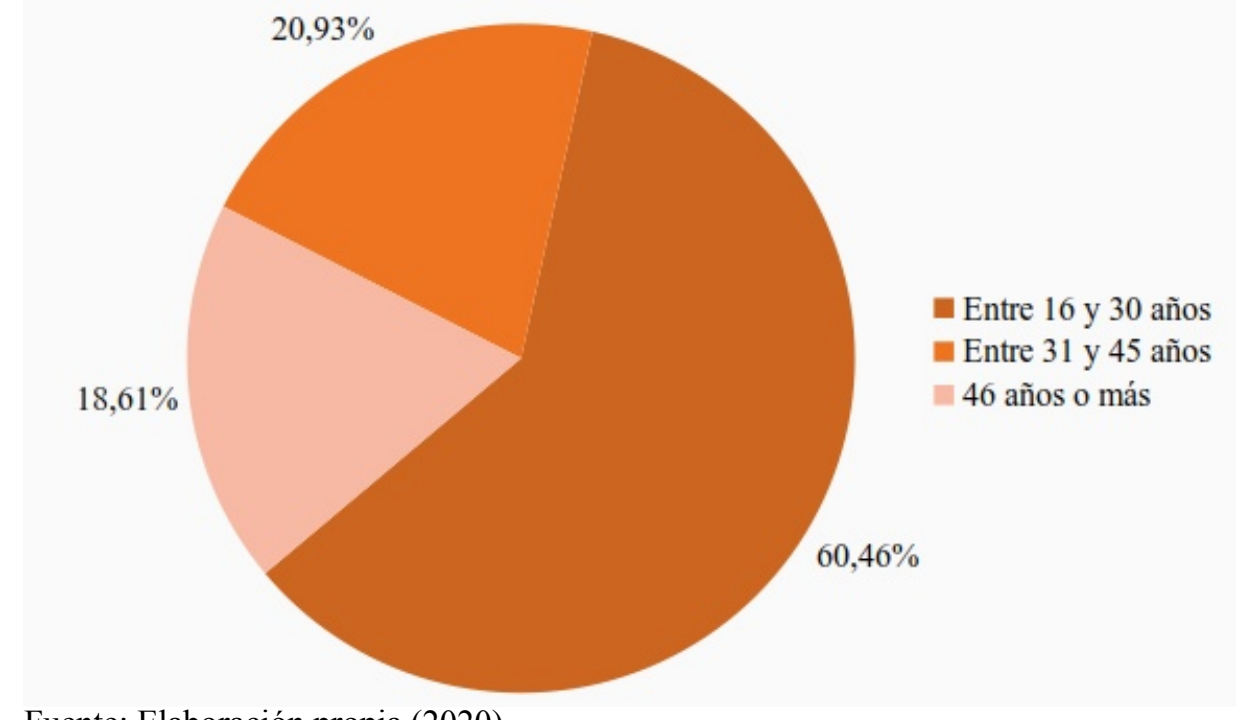

Fuente: Elaboración propia (2020)

Figura 2: Datos demográficos (provincia de origen)

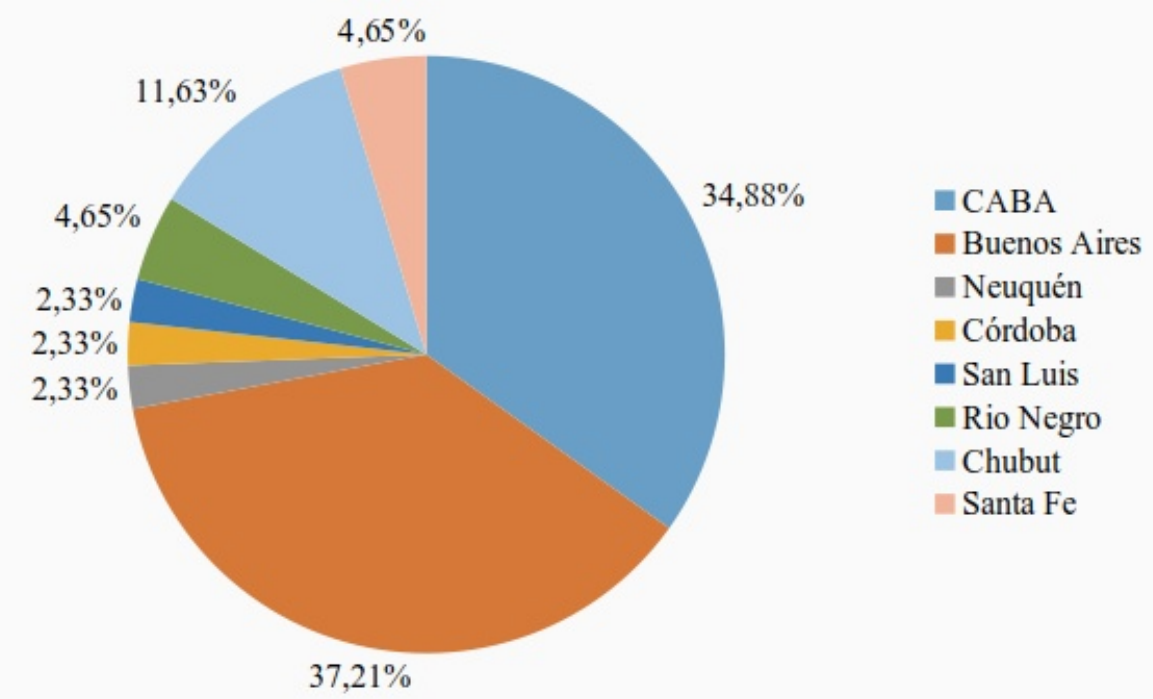

Fuente: Elaboración propia (2020)

La pregunta 1 apuntó a identificar qué tipo de definición elegían las participantes como la más adecuada para conceptualizar el acoso sexual callejero, pudiendo combinarse opciones. 
Representaciones Sociales sobre Acoso Sexual Callejero en Mujeres

Activistas Participantes del XXXIII Encuentro Nacional de Mujeres, Argentina

Las respuestas preestablecidas fueron:

a) Gritos, silbidos o señas sexuales dirigidos a una mujer desconocida en cualquier espacio público o de acceso público sin motivo.

b) Cualquier acto con connotación sexual que busca degradar, intimidar y/o atemorizar a la mujer en cualquier espacio público o de acceso público.

c) Un acto de poder motivado por la jerarquía de género que busca degradar, intimidar y/o atemorizar a una mujer.

d) Ninguna de las anteriores.

Del total de las participantes, 36,36\% eligió la respuesta b); 29,55 eligió combinar las respuestas a), b) y c); 20,45\% seleccionó la respuesta c); 6,82\% combinó las opciones b) y c); 4,55\% consideró que ninguna opción era correcta; y 2,27\% señaló las respuestas a) y b). La respuesta a) y la combinación de las respuestas a) y c) no fue seleccionada por ninguna de las participantes (Figura 3).

Figura 3: Distribución de la selección de respuestas a la pregunta 1

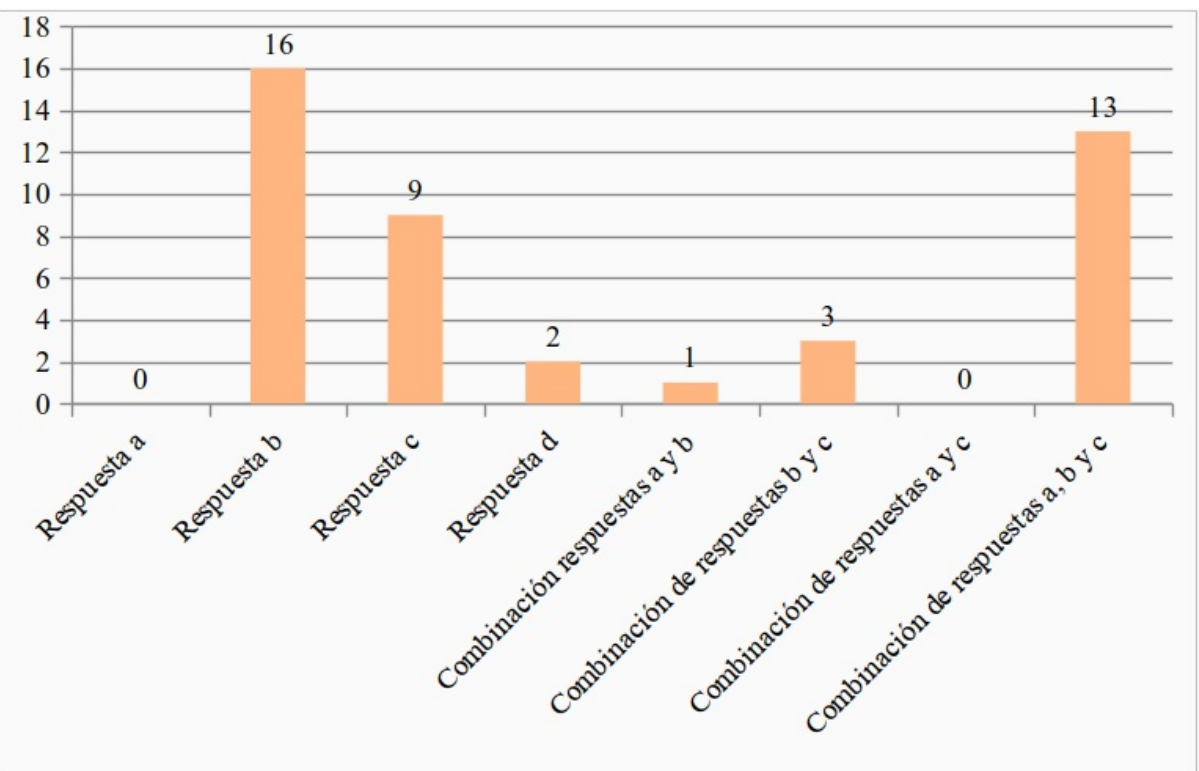

Fuente: Elaboración propia (2020)

La pregunta 2 interpeló a las participantes a contestar dicotómicamente entre "Sí" o "No" ante la afirmación "el acoso sexual callejero es un problema de seguridad", optando $86,37 \%$ por la afirmativa (Figura 4).

Figura4: Distribución de la selección de las respuestas a la pregunta 2

PREGUNTA

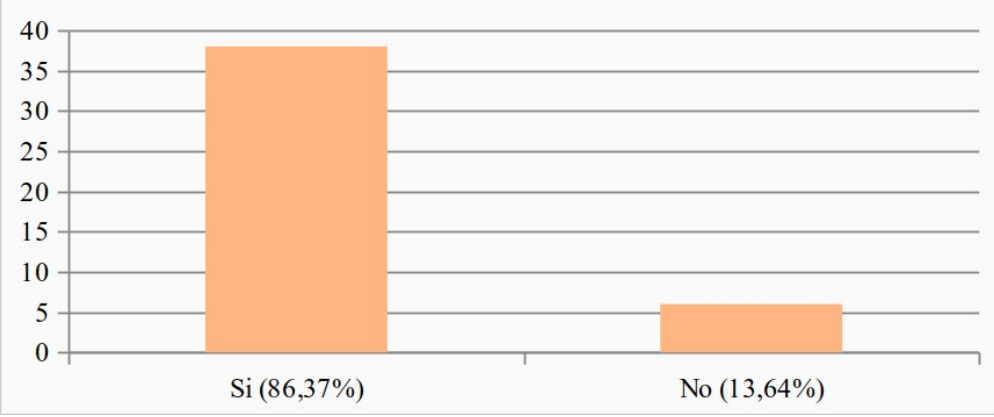

Fuente: Elaboración propia (2020) 
Representaciones Sociales sobre Acoso Sexual Callejero en Mujeres

Activistas Participantes del XXXIII Encuentro Nacional de Mujeres, Argentina

La pregunta 3 consultó respecto de la necesidad de intervención del Estado frente al acoso sexual callejero, debiendo las participantes contestar dicotómicamente entre "Sí" o "No". El 100\% de las mujeres respondieron por la afirmativa.

La pregunta 4 debía ser contestada sólo si la anterior fue respondida afirmativamente por lo que, dados los resultados, la totalidad de las participantes debieron seleccionar una o más opciones preestablecidas, las cuales fueron: delito.

a) Establecer en el Código Penal que el acoso sexual callejero es un

b) Que cada Estado provincial establezca que el acoso sexual callejero es una contravención.

c) Reforzar las campañas en contra del acoso sexual callejero.

d) Implementar la Educación Sexual Integral.

Del total, 27,27\% combinó las respuestas a), b), c) y d); el 13,64\% puso en juego las opciones b), c) y d); 11,36\% las respuestas a), c) y d); mismo resultado arrojó la combinación de las respuestas c) y d); 6,82\% combinó las opciones a) y d); 4,55\% seleccionó la respuesta d); mismo porcentaje para la combinación de respuestas a) y b); y 2,27\% seleccionaron independientemente las respuestas b) y c). Ninguna de las participantes seleccionó la combinación de las respuestas b y c), a) y c), a), b) y c) ni a), b) y d) (Figura 5).

Figura 5: Distribución de la selección de las respuestas a la pregunta 4

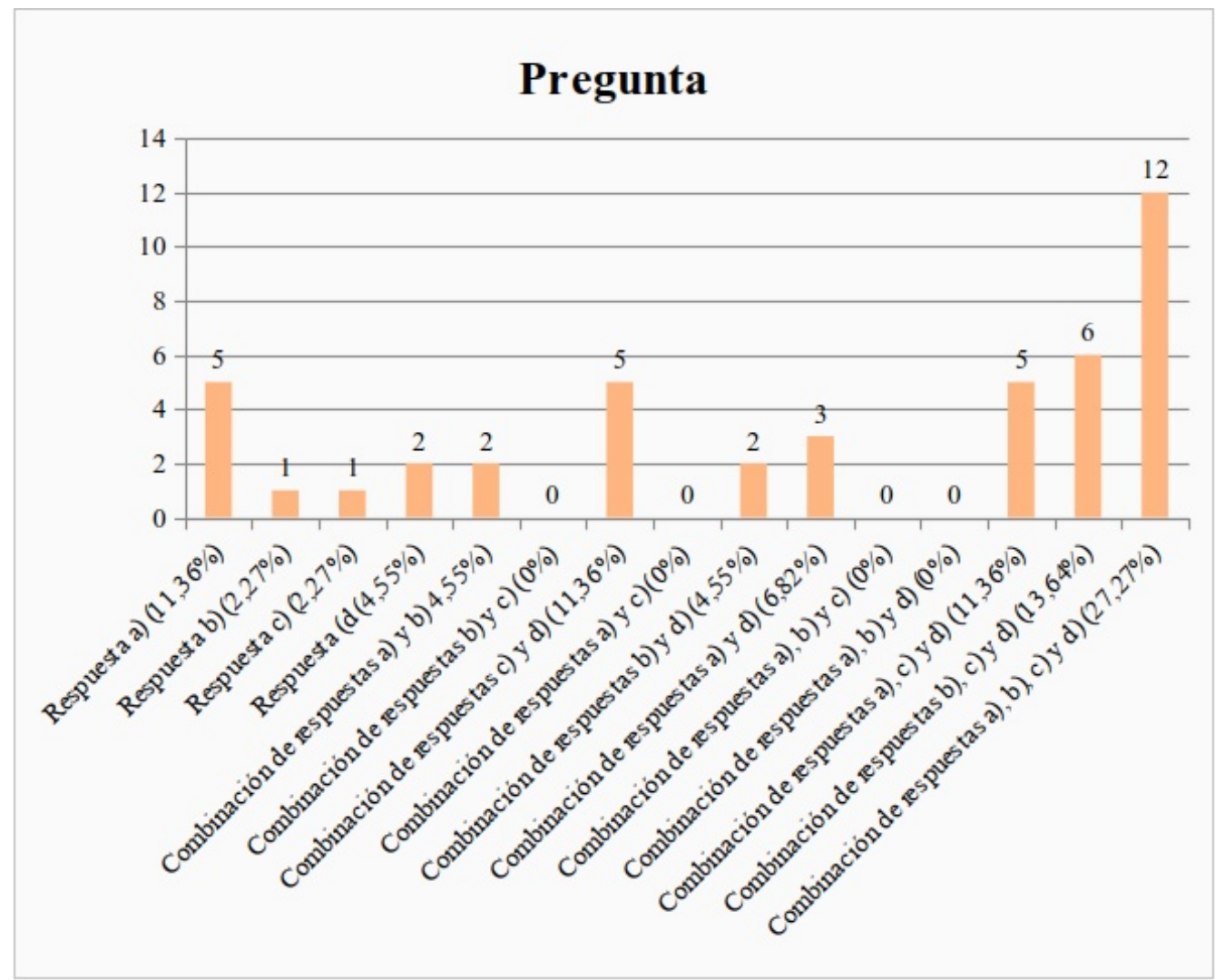

Fuente: Elaboración propia (2020) 
Representaciones Sociales sobre Acoso Sexual Callejero en Mujeres

Activistas Participantes del XXXIII Encuentro Nacional de Mujeres, Argentina

Finalmente, si las participantes seleccionaban las opciones a) y/o b), tanto individualmente como en combinación con otra/s respuestas, debían pasar a contestar la pregunta 5, que apuntó a la sanción a imponerse a quien comete un acto de acoso sexual callejero. Las respuestas podían combinarse, y estuvieron preestablecidas de la siguiente manera:
a) Prisión
b) Multa
c) Trabajo comunitario
d) Curso de concientización sobre la problemática

Del total, el 22,73\% optó por combinar las respuestas c) y d); $13,64 \%$ seleccionó la respuesta d); 11,36\% la respuesta a); mismo porcentaje para la combinación de respuestas a) y d); 9,09\% combinaron las opciones b), c) y d); $6,82 \%$ combinaron las respuestas b) y d); $4,54 \%$ combinaron las opciones b) y c); mismo porcentaje para la combinación de a), b), c) y d); 2,72\% optaron por la respuesta c); mientras que nadie seleccionó la respuesta b) ni las combinaciones a) y b); a) y c); a), b) y c); ni a), c) y d) (Figura 6).

Figura 6: Distribución de la selección de las respuestas a la pregunta 4

\section{Pregunta}

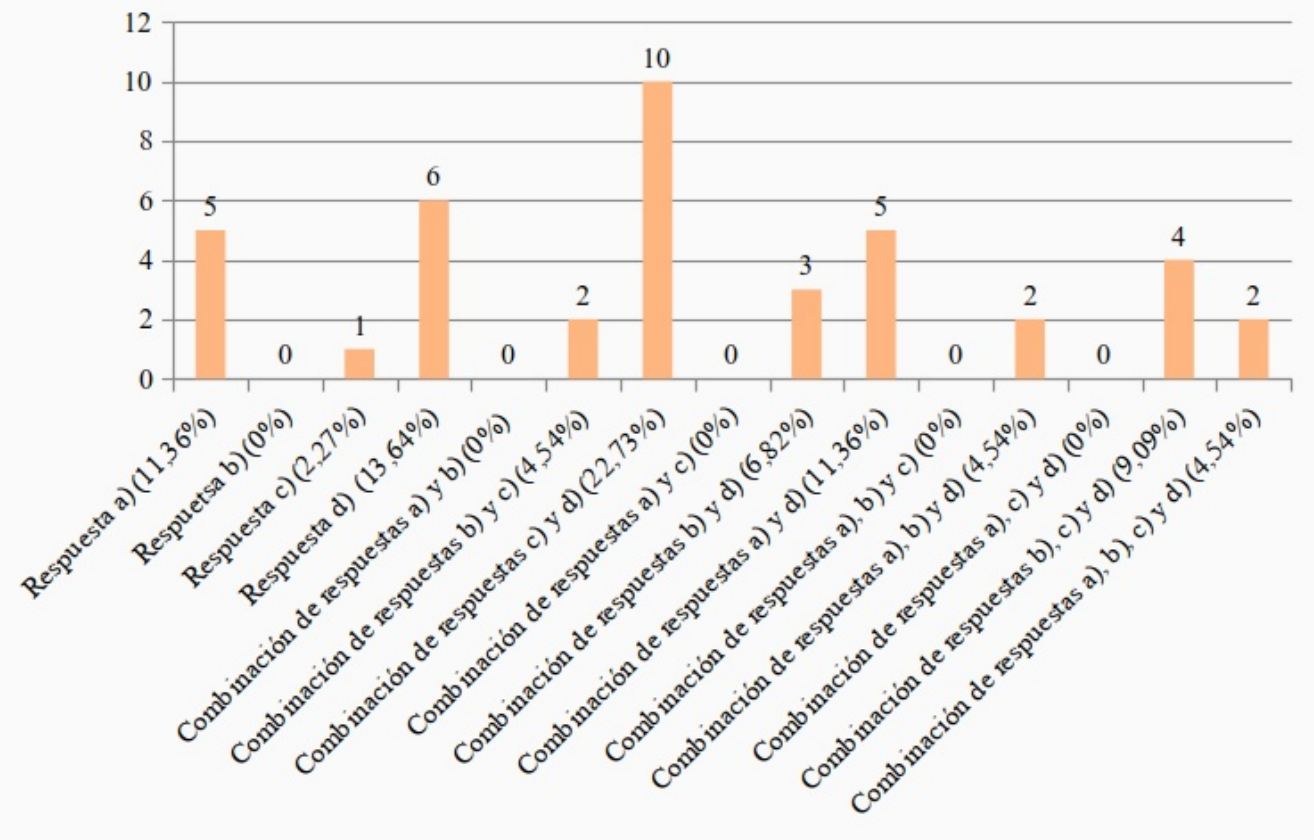

Fuente: Elaboración propia (2020)

\section{Discusión y conclusiones}

Este estudio busca abordar las representaciones sociales vinculadas al acoso sexual callejero en una población particular, que en este caso consistió en mujeres políticamente involucradas en el debate acerca de la construcción, acceso y goce de la ciudad y el hábitat, lo cual las motivó a participar en el Taller 43 "Mujeres y Hábitat" del XXXIII Encuentro Nacional de Mujeres (2018). Si bien la muestra no es representativa, la importancia de este trabajo 


\section{Activistas Participantes del XXXIII Encuentro Nacional de Mujeres, Argentina}

radica en que en este taller es donde las mujeres coordinan una agenda nacional unificada de cara a las nuevas luchas vinculadas a la temática, llevando a la comisión posiciones consensuadas en el interior de las organizaciones de las cuales participan, o bien sus opiniones personales, en caso de concurrir independientemente.

Respecto a las representaciones sociales sobre qué es el acoso sexual callejero como fenómeno, en el cuestionario suministrado se pretendió reflejar dos tipos de definiciones: 1) una que en este trabajo recibirá el nombre de "definición situacional", por centrar como elementos conceptuales estructurales a acciones individualizadas del victimario y al espacio en el cual la agresión tiene lugar [respuesta a) a la pregunta 1] ; y 2) otra que se llamará "definición coyuntural/relacional", por ser los rasgos relevantes para definir al acoso sexual callejero la desigualdad en las relaciones basadas en género y sus consecuencias en los procesos de producción de la ciudad en general y de los espacios públicos en particular [respuestas b) y c) a la pregunta 1]. De la comparación de los datos obtenidos surge que 93,18\% de las respuestas brindadas se corresponden con definiciones de tipo coyuntural/relacional (Figura 7), lo cual coincide con conceptos como el que afirman que:

El acoso sexual de mujeres es una manifestación de una cultura machista, que persiste en nuestra sociedad y que aún no es sancionada ética $\mathrm{y}$ legalmente, hecho que ha posibilitado que estas manifestaciones sean toleradas y perpetuadas como comportamientos normales, lo que hace que las mujeres estén sometidas en un proceso de cosificación, despojándoles de todo derecho como persona humana" (MEDINA et al, 2016, p. 62).

Figura 7: Distribución de las preferencias conceptuales

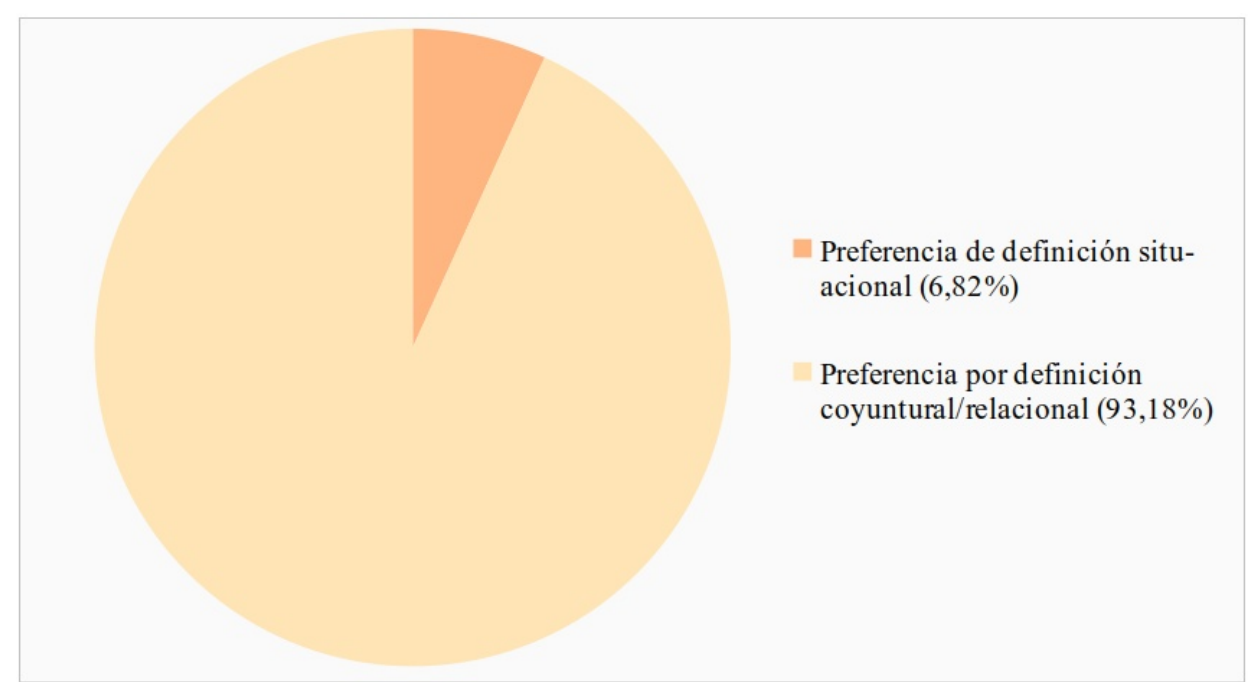

Fuente: Elaboración propia (2020).

En este sentido, el acoso sexual callejero adquiere también una función de control social difuso (es decir, no estatizado) sobre los cuerpos de las mujeres que consiste en "un ejercicio de poder sobre la autonomía personal y el libre albedrío" (GALVAÑO TER-AKOPIAN, 2020, p. 8).

En lo relativo a la cualidad de la intervención estatal y el tipo de sanción 
merecida por el agresor, el análisis comparativo se vuelve interesante si la comprendemos desde la actual coyuntura, en la que funcionarios y medios de comunicación hacen uso de históricas luchas de las mujeres para justificar políticas de corte punitivo. Un $50 \%$ de las respuestas contenían como preferencia a la reacción punitiva como intervención predilecta (Figura 8), pero sólo $28,57 \%$ de las opciones elegidas contemplaron la prisión como sanción al victimario (Figura 9).

Figura 8: Distribución de las preferencias respecto de la forma de intervención estatal

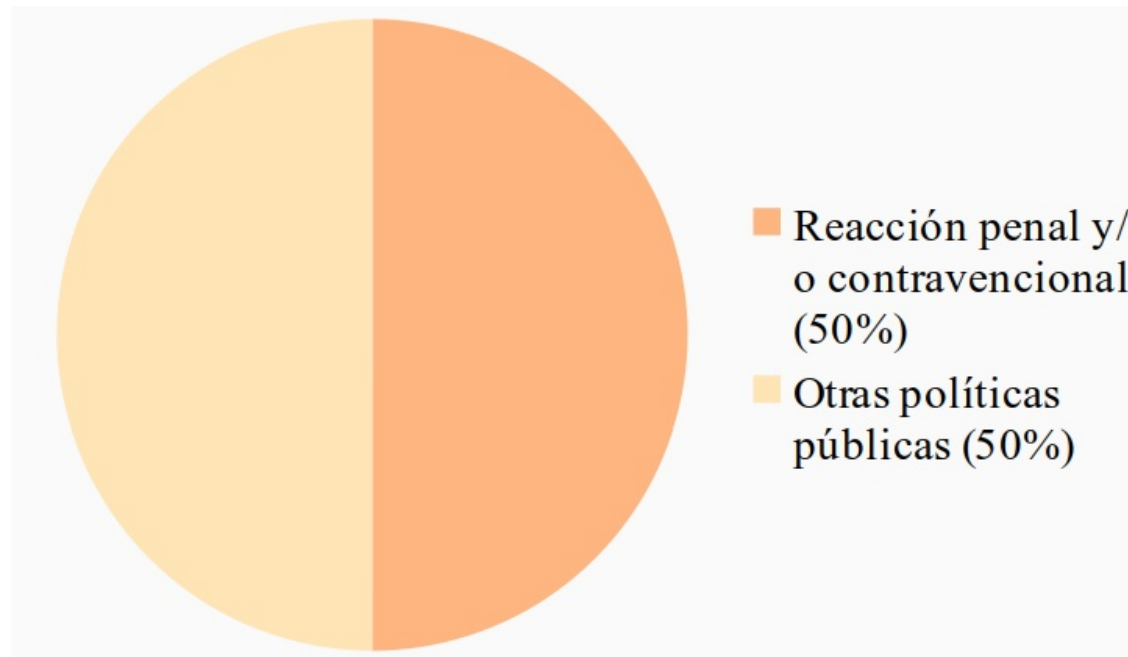

Fuente: Elaboración propia (2020).

Figura 9: Distribución de las preferencias respecto de la sanción a quien comete un acto de acoso sexual callejeros

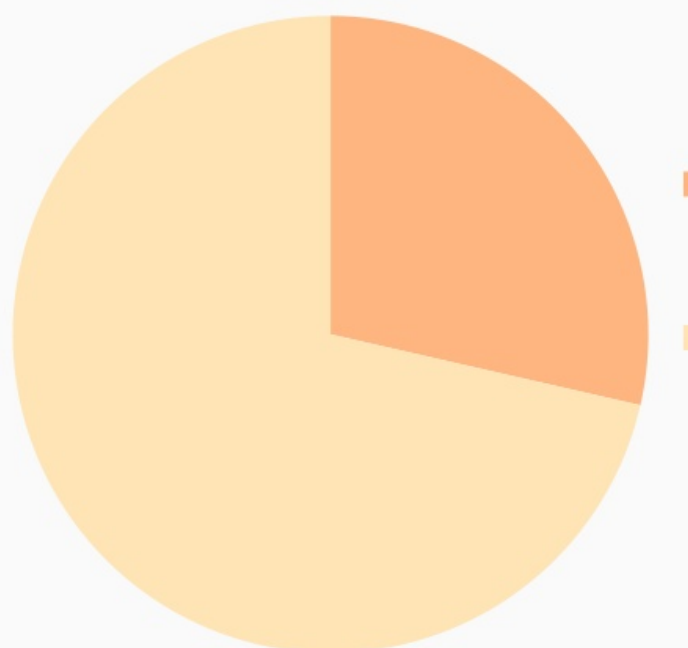

Total elección de la sanción de prisión $28,57 \%$ )

Total elección de sanción con medidas alternativas $(71,43 \%)$

Fuente: Elaboración propia (2020).

Estos resultados son similares a los obtenidos por la Dirección General de la Mujer dependiente del Ministerio de Desarrollo Social de CABA, organismo que, previo a la tipificación contravencional del acoso sexual callejero, realizó una encuesta general en la que los entrevistados prefirieron una actuación del Estado por fuera de la esfera penal en sentido estricto y, en caso de solicitar sanciones a los victimarios, optaron por medidas alternativas a la prisión. No obstante, a esta postura social, la ley 5742 prefirió convertir el acoso sexual 
callejero en contravención (ALADRO, 2017a).

A partir de este antecedente, se realizó un rastreo normativo para poner en discusión estos resultados con legislación vigente y proyectada en las provincias de origen de las encuestadas (Tabla 1). Del relevamiento legislativo surge la predominancia de posibles normas aplicables no específicas, es decir, que si bien no se tipifica el acoso sexual callejero explícitamente existen otros tipos contravencionales a través de los cuales puede sancionarse este tipo de conducta violenta contra las mujeres. A la vez, los legisladores que han tipificado especialmente al acoso sexual callejero, consideraron que la respuesta más adecuada eran las medidas alternativas a la prisión, reservándose el arresto sólo para casos extremos.

Tabla 1: Legislación vigente y proyectos de ley de tipificación contravencional del acoso sexual callejero en las provincias de origen de las mujeres participantes del estudio

\begin{tabular}{|c|c|c|c|c|}
\hline & $\begin{array}{c}\text { Legislación } \\
\text { vigente }\end{array}$ & $\begin{array}{l}\text { Sanción } \\
\text { vigente }\end{array}$ & $\begin{array}{l}\text { Proyectos de } \\
\text { reforma }\end{array}$ & Sanción proyectada \\
\hline $\mathrm{CABA}$ & $\begin{array}{l}\text { Artículo } 65 \text { bis del } \\
\text { Código } \\
\text { Contravencional } \\
\text { (Ley 5742): "Acoso } \\
\text { sexual en espacios } \\
\text { públicos o de acceso } \\
\text { público. } \\
\text { acosare sexualmente } \\
\text { a otro, en lugares } \\
\text { públicos o privados } \\
\text { de acceso público, } \\
\text { siempre que el hecho } \\
\text { no constituya delito". }\end{array}$ & 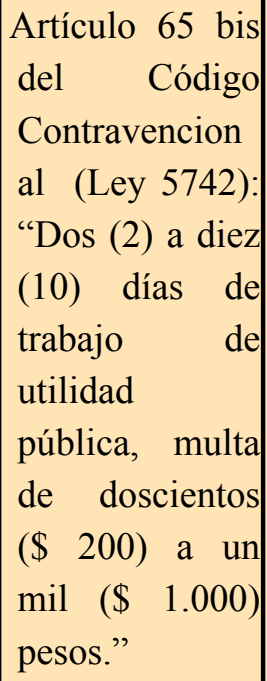 & - & - \\
\hline $\begin{array}{l}\text { Buenos } \\
\text { Aires }\end{array}$ & - & - & $\begin{array}{l}\text { PROYECTO DE } \\
\text { LEY D } 622 \quad 2017 \text { - } \\
2018 \\
\text { Artículo70 } \\
\text { bis“(...)quien acosare } \\
\text { sexualmente a otro en } \\
\text { lugares públicos o } \\
\text { privados de acceso } \\
\text { público siempre que } \\
\text { el hecho no constituya } \\
\text { delito.” }\end{array}$ & $\begin{array}{l}\text { PROYECTO DE } \\
\text { LEY D } 622 \text { 2017 } \\
2018 \\
\text { "Artículo } 70 \text { bis. } \\
\text { Será penado con } \\
\text { multa entre el veinte } \\
\text { (20) y el cuarenta (40) } \\
\text { por ciento del haber } \\
\text { mensual del Agente } \\
\text { de Seguridad } \\
\text { (Agrupamiento } \\
\text { Comando) de la } \\
\text { Policía de la provincia } \\
\text { de Buenos Aires(...)" }\end{array}$ \\
\hline
\end{tabular}


Representaciones Sociales sobre Acoso Sexual Callejero en Mujeres

Activistas Participantes del XXXIII Encuentro Nacional de Mujeres, Argentina

\begin{tabular}{|c|c|c|c|c|}
\hline San Luis & - & - & Sin registro & Sin registro \\
\hline Santa Fe & $\begin{array}{c}\text { Artículo } 48 \text { del } \\
\text { Código de } \\
\text { Convivencia: “Acoso } \\
\text { Sexual Callejero. } \\
\text { Será punible el que } \\
\text { hostigue mediante } \\
\text { todo acto de } \\
\text { naturaleza o } \\
\text { connotación sexual, } \\
\text { cometido en contra } \\
\text { de una o varias } \\
\text { personas en espacios } \\
\text { públicos o espacio } \\
\text { privado de acceso } \\
\text { público, sin que } \\
\text { medie el } \\
\text { consentimiento de } \\
\text { la víctima, } \\
\text { produciendo en la } \\
\text { misma intimidación, } \\
\text { hostilidad, } \\
\text { degradación, } \\
\text { humillación, o un } \\
\text { ambiente ofensivo en } \\
\text { los espacios públicos. }\end{array}$ & 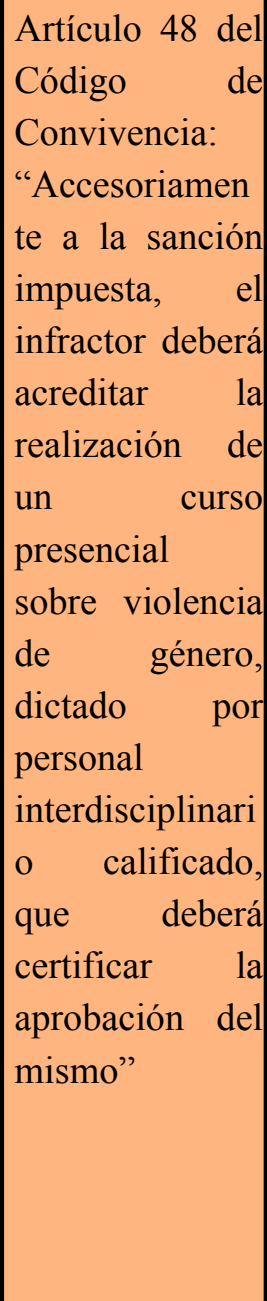 & - & - \\
\hline Chubut & - & - & $\begin{array}{c}\text { Proyecto de Ley n } \\
\text { 018/16, por el cual se } \\
\text { instituye la Ley de } \\
\text { Abordaje Integral y } \\
\text { Lucha Contra la } \\
\text { Violencia, } \\
\text { Hostigamiento y } \\
\text { Acoso hacia la Mujer } \\
\text { (Único registro } \\
\text { disponible: pase a } \\
\text { Comisión de } \\
\text { Legislación Social, } \\
\text { Salud, Derechos } \\
\text { Humanos y Trabajo) }\end{array}$ & Sin registro \\
\hline
\end{tabular}




\begin{tabular}{|c|c|c|c|c|}
\hline Córdoba & - & - & 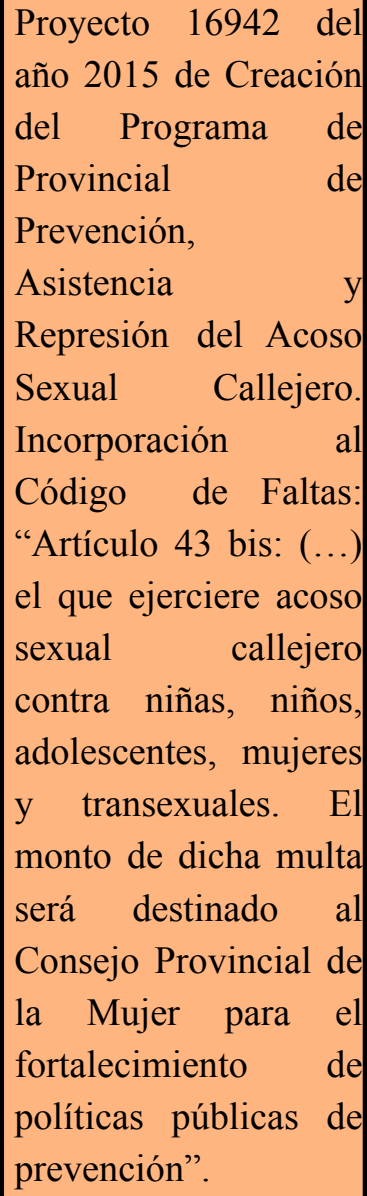 & $\begin{array}{l}\text { Proyecto } 16942 \\
\text { año } 2015 \text { de } \\
\text { del Preación } \\
\text { Provincial } \\
\text { Prevención, } \\
\text { Asistencia } \\
\text { Represión del Acoso } \\
\text { Sexual Callejero. } \\
\text { Incorporación } \\
\text { Código de Faltas: } \\
\text { "Artículo } 43 \text { bis: (...) } \\
\text { Será penado con hasta } \\
\text { cinco (5) días de } \\
\text { trabajo comunitario, } \\
\text { multa de hasta diez } \\
\text { Unidades de Multa } \\
(10 \text { UM) o arresto de } \\
\text { hasta tres (3) días } \\
\text { (...)" }\end{array}$ \\
\hline Neuquén & - & - & 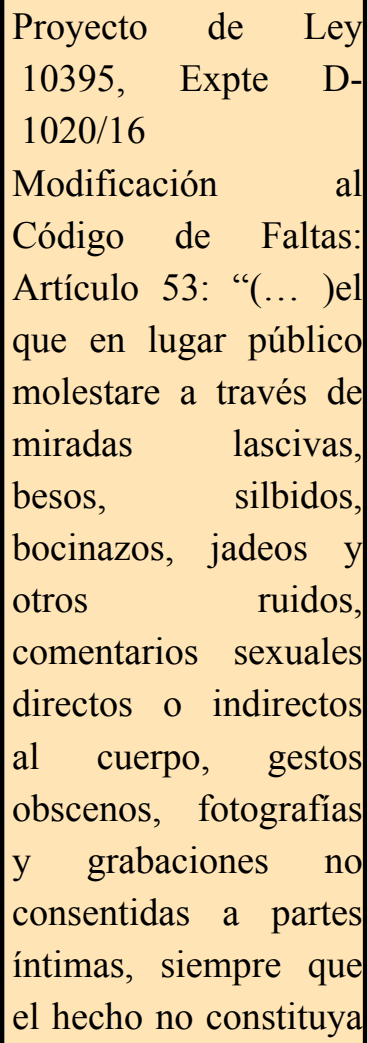 & $\begin{array}{l}\text { Proyecto de } r \text { Ley } \\
10395, \text { Expte } \\
1020 / 16 \\
\text { Modificación }\end{array}$ \\
\hline
\end{tabular}




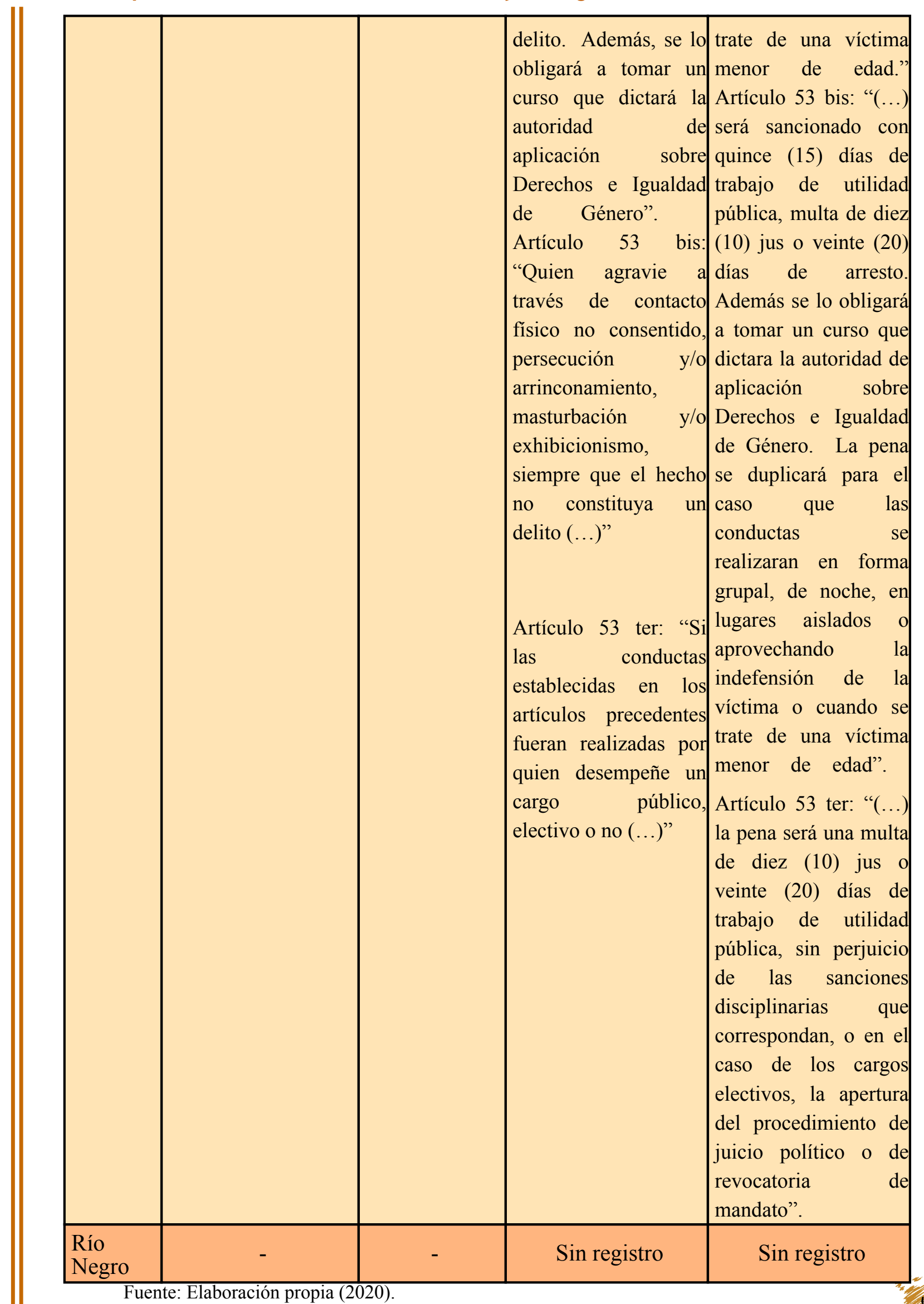

Almendra Aladro 
Por otro lado, los tipos contravencionales excluyen, en tanto su característica cerrada, definiciones como la coyuntural/relacional de la que se ha dado cuenta en este trabajo y, por tanto, son deficientes para dar solución adecuada a la problemática. Además, si se afirma la superposición de funciones entre el Derecho Contravencional y el Derecho Penal (ZAFFARONI et al., 2000), y se acepta que el primero se rige por los mismos principios que el segundo, pero se ejerce con mayor nivel de arbitrariedad, suponiendo que se persiga el objetivo de disuadir a los acosadores, ¿resulta realmente eficaz aplicar sanciones a este tipo de conductas? Y si a esa arbitrariedad se suma al histórico maltrato y revictimización que sufren las mujeres al intentar ejercer su derecho de acceso a la justicia, ¿brinda una auténtica respuesta a la víctima desde una perspectiva de derechos?

El acoso sexual callejero es una forma de violencia que se desarrolla en contextos urbanos, la cual cada mujer vivirá a lo largo de su vida unas 328500 veces con distintos grados de gravedad y afectación. Pero que, en definitiva, repercuten en el desarrollo de su vida cotidiana generando un daño que Greta Galvaño Ter-Akopian denomina "estrés callejero" y que define como "un estado de alerta constante por no poder caminar libremente sin sufrir acoso callejero" (GALVAÑO TER-AKOPIAN, 2020). Estos datos delatan, además, que esta forma de violencia cuenta con una extensión de gigantescas magnitudes y que requiere modelos de intervención radicales, es decir, que den cuenta de su origen, características y consecuencias.

Hasta el momento, el ordenamiento jurídico argentino ha reconocido el acoso sexual callejero como una modalidad de la violencia de género en la Ley 26428 y la Cámara de Diputados ha dado media sanción en 2019 al proyecto de ley que busca incorporarlo al Código Penal de la Nación (a esta segunda acción se la llamará aquí "modelo de intervención penal"), mientras que las provincias en el ejercicio de sus competencias han determinado localmente qué consideran acoso sexual callejero, cuál es su sanción y cuáles son los pasos a seguir desde el momento del hecho hasta la obtención de una respuesta jurisdiccional (esta estrategia será aquí denominada "modelo de intervención contravencional").

A estos modelos de intervención se sumará a esta discusión un tercero, que en este artículo se designará como "modelo de daños", que construye a partir de las soluciones dadas a dos casos ocurridos en Resistencia (provincia de Chacho) en 2018 y en Ciudad Autónoma de Buenos Aires en 2019. En el primero de ellos, el Jzagado Laboral $\mathrm{N}^{\circ} 4$ reconoció en la causa "G., J. A y Otro c/ T. Hnos. SRL s/Despido" que fue justo el despido de dos empleados que durante sus labores agredieron verbalmente a una mujer que transitaba por la vía pública; "en tanto debe tenerse en cuenta la gravedad de las palabras contra la dignidad de la denunciante (...) un hecho que hoy adquiere gravedad superlativa". En el segundo caso, la abogada Greta Galvaño Ter-Akopián instó una acción civil por daños y perjuicios a una empresa que empleaba a personas que la acosaban en horario laboral y en instalaciones de la empresa cuando ella pasaba por el lugar caminando, y logró un acuerdo en mediación civil prejudicial histórico ${ }^{3}$.

A partir de estos casos, el modelo de daños como estrategia frente al acoso sexual callejero implica reconocer sus efectos en las mujeres como hechos 
jurídicamente relevantes y, en el último caso, como daño moral, es decir, como "el menoscabo que sufre una persona en su bienestar psíquico (espiritual) sin que el estado negativo sobreviniente (tristeza, dolor, amargura, inseguridad, angustia, etc.) llegue a configurar una situación patológica" (ALFERILLO, 2020:5).

Esta estrategia, que abandona la idea de la sanción y la reemplaza por la reparación de la víctima, cuenta con la ventaja de no requerir una tipificación legal de acciones de tipo cerrada, sino que, al regirse por las normas del derecho civil, bastará con la prueba del hecho a los fines de obtener el resarcimiento. No obstante esta ventaja, la justicia civil en Argentina requiere para este tipo de acciones el patrocinio letrado, lo cual podría implicar un menoscabo a su derecho al acceso a la justicia si el Estado no asegura la representación de las mujeres que acudan a esta instancia.

El modelo de daños presenta la potencialidad, a diferencia de los modelos de intervención penal y contravencional, de ser objeto de procesos restaurativos que impliquen a la comunidad en su conjunto. Siendo el acoso sexual callejero una forma de violencia que se desarrolla en contextos urbanos, la característica espacial se torna clave para comprenderlo como ejercicio de poder sobre las mujeres que ocasiona que el espacio sea injustamente distribuido para ellas en relación a los varones, es decir, que el acoso sexual callejero es una de las consecuencias y, dialécticamente, uno de los hechos que caracteriza al proceso geográfico injusto y discriminatorio por motivos de género (SOJA, 2014).

Así, frente a las políticas de extrema securitización y "mano dura" que impregnan las ciudades y que, en la actualidad, echan mano de los reclamos históricos de las mujeres para formar una lógica justificadora de estas estrategias represivas, dentro de las cuales podrá tomarse el acoso sexual callejero. La idea de "justicia espacial" de Edward Soja puede implicar a las comunidades en procesos de mediación (RODRÍGUEZ FERNÁNDEZ, 2008) en la resolución de los problemas que se desarrollan en la vida cotidiana en general y, en particular, en el resarcimiento del daño que esta modalidad de la violencia de género produce en las mujeres desde una lógica de fuerte participación ciudadana.

Lejos de pretender concluir el debate de los resultados aquí traídos, este trabajo pretende constituirse como un aporte al estímulo de la producción de artículos científicos que permitan orientar el futuro diseño, ejecución $y$ evaluación en materia de acoso sexual callejero, entendiendo que las mujeres deben ser escuchadas tanto a la hora de la toma de decisiones relativas a la producción de la ciudad, de la cual son parte como al momento de resolver situaciones como lo constituyen los hechos de acoso sexual callejero que afectan sus vidas cotidianas.

3 Greta Galvaño Ter-Akopián es una abogada que reside en Ciudad Autónoma de Buenos Aires y fundó Libres de Acoso Callejero (LIDA) con el propósito de visibilizar la problemática. Las generalidades de su caso pueden consultarse en:

https://www.lanacion.com.ar/sociedad/denuncio-local-comidas-palermo-acoso-sexualcallejero-nid2318106. 


\section{Bibliografía}

ALADRO, A, Debates feministas en torno al Derecho al Habitat Principales reclamos emanados del Encuentro Nacional de Mujeres, III Congreso de Estudios Poscoloniales y IV Jornadas de Feminismo Poscolonial "Interrupciones desde el Sur: habitando cuerpos, territorios y saberes", Buenos Aires, 2016.

ALADRO, A. (a). Mujeres en la ciudad: El acoso callejero en la Ciudad Autónoma de Buenos Aires. Jornadas. Desafíos actuales de la Justicia porteña: Autonomía e Igualdad. Facultad de Derecho, Universidad de Buenos Aires. 2017.2 Disponible $<$ http://www.adaciudad.org.ar/docs/AladroMujeres-en-la-ciudad.-El-acosocallejero-en-laCiudad-Aut $\% \mathrm{C} 3 \% \mathrm{~B} 3$ noma-de-Buenos-Aires.pdf $>$

ALADRO, A. (b), El Encuentro Nacional de Mujeres como espacio de construcción del Derecho a la Ciudad desde la perspectiva de género, Adelantos de Investigación en Ciencias Jurídicas y Sociales, Mar del Plata: CIDDH-UNMdP, 2017.

ALFERILlO, P, E., Cuantificación del daño moral en el Código Civil y Comercial. RCyS, v. 4, n. 3, p. 1 - 23, 2020.

ALMA, A. y LORENZO, P. Mujeres que se encuentran. Una recuperación histórica de los Encuentros Nacionales de Mujeres en Argentina (19862005). Buenos Aires: Feminaria Editora, 2009.

ANGILLETA, M. F, Encuentros nacionales de mujeres (1986 hasta el presente). Los derechos de las mujeres. Pensar la Democracia. Treinta ejercicios para trabajar en el aula. Lugar: Buenos Aires, 2013.

CORAZÓN LLERENA BENITES, R., Percepción y actitudes frente al acoso sexual callejero en estudiantes mujeres de una Universidad Privada de Medicina, Horizonte Médico, v. 16, n. 1, p. 62 -68, 2016.

FALÚ, A. La omisión del género en el pensamiento de las ciudades. In: BORJA, J.; CARRIÓN, F.; CORTI, M. (de) Ciudades para cambiar la vida: Una respuesta a Hábitat III. Ciudad Autónoma de Buenos Aires: Café de las Ciudades, 2016, p. 159 - 169.

FALÚ, A., La fuerza estigmatizadora del acoso sexual: violencias en el transporte público. Revista Vivienda y Ciudad , v. 4, p. 205 - 208, 2017.

FELITTI, K.; ¿Qué entienden los feminismos por laicidad? Consignas, debates y acciones en los Encuentros Nacionales de Mujeres (2015-2017). IX Jornadas " $L a$ institución como proceso: configuraciones de lo religioso en las sociedades contemporáneas", Buenos Aires, 2017. 
FERNÁNDEZ VÁZQUEZ, S. S, Los Encuentros Nacionales de Mujeres: Foros Deliberativos en la construcción de la agenda política de género en la Argentina. Mujeres iberoamericanas y derechos humanos. Estrategias feministas, acción políticas y exilios, Sevilla, España: Paidós, 2016.

GALVAÑO TER-AKOPIÁN, G., Marco legal y jurisprudencial del acoso callejero en el ámbito de la Ciudad Autónoma de Buenos Aires. Pensar Decir Hacer, n², Ciudad Autónoma de Buenos Aires, Argentina:SITRAJU, pp. 4-8, 2020.

GARCÍA, G. I., La mujer decide, la sociedad respeta, ¿el Estado garantiza? Los Encuentros Nacionales de Mujeres. Revista Dos puntas, n. 7, p. 113 129, 2013.

HERRERA, M. I. Apuntes para interpretar el crecimiento de los Encuentros Nacionales de Mujeres en las luchas colectivas en Argentina. Revista Utopías, n. ${ }^{\circ} 23$, p. $1-10,2017$.

MARTYNOWSKYJ, E., Prostitución y feminismo(s). Disputas por el reconocimiento en los Encuentros Nacionales de Mujeres (Argentina, 19862016). Seminario Internacional Fazendo Gênero 11- Transformacoes, conexoes, deslocamentos. Brasil, Florianopolis, 2017.

MEDINA VILCA, G; ZAPANA CASTRO, A., Representaciones sociales de las mujeres jóvenes sobre el acoso sexual callejero en la ciudad de Puno. Punto Cero, v. 21, n. 33, p. 60 - 81, 2016.

MORALES ALFONSO,L; QUIEOZ DEL POZO, N; y RAMÍREZ IGLESIAS, G., Acoso sexual en lugares públicos de Quito: retos para una "ciudad segura. Revista Latinoamericana de Estudios de Seguridad, n. 19, p. 21 - 36, 2016.

LEFEBVRE, H., El derecho a la ciudad. París, Francia:Ediciones Península, 1968.

RODRÍGUEZ FERNÁNDEZ, G., ¿Comunidad? Mediación comunitaria, habitar efímero y diversidad cultural. Revista Polis, p. 87 - 203, 2008.

SCIORTINO, S., Procesos de organización política de las mujeres indígenas en el movimiento amplio de mujeres en Argentina. Consideraciones sobre el feminismo desde la perspectiva indígena. Universitas humanística, n. 79, p. $65-87,2015$.

SCIORTINO, S., Políticas de Identidad en los Encuentros Nacionales de Mujeres (Argentina): momentos coyunturales en la conformación de una agenda política; Revista Vía Iuris, p. 89 - 108, 2017.

SOJA, E., En busca de la justicia espacial, Valencia, España:Tirand 
Representaciones Sociales sobre Acoso Sexual Callejero en Mujeres

Activistas Participantes del XXXIII Encuentro Nacional de Mujeres, Argentina

Humanidades, 2014.

VILLARROEL, G., Las representaciones sociales: una nueva relación entre el individuo y la sociedad. Fermentum: Revista Venezolana de Sociología y Antropología, v. 17, n. 49, p. 434 - 354, 2007.

ZAFFARONI, E., AlAGiA, A. y SLOKAR, A., Derecho Penal: Parte general, Buenos Aires: Ediar, 2000. 